\title{
Fracture-arrachement de la tubérosité tibiale antérieure chez un jeune sportif
}

\author{
Avulsion fracture of the anterior tibial tuberosity in a young athlete
}

\author{
D. Tourdias $\cdot$ V. Hostyn $\cdot$ G. Tourdias
}

Reçu le 14 octobre 2013 ; accepté le 9 décembre 2013

(C) SFMU et Springer-Verlag France 2014

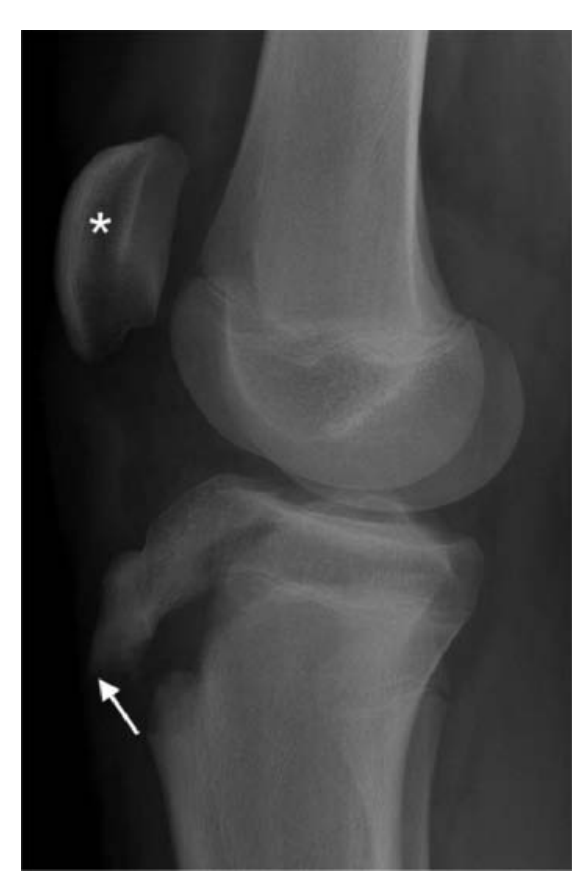

Fig. 1 Fracture-arrachement de la tubérosité tibiale antérieure (flèche) se prolongeant à travers l'épiphyse tibiale proximale (type IIIA de la classification de Ogden) avec patella alta secondaire (astérisque) signant la rupture complète de l'appareil extenseur

D. Tourdias $(\bowtie) \cdot$ V. Hostyn

Service d'accueil des urgences-Smur,

centre hospitalier Sud Gironde, rue Paul Langevin,

F-33210 Langon, France

e-mail : tourdiasdamien@yahoo.fr

\section{G. Tourdias}

Service de chirurgie orthopédique et traumatologique, centre hospitalier Sud Gironde, rue Paul Langevin,

F-33210 Langon, France
Un patient de 15 ans se présente aux urgences pour une douleur aiguë du genou gauche suite à un traumatisme indirect survenu quelques heures auparavant au cours d'un match de handball. Cette vive douleur, associée à un craquement, est apparue brutalement lors d'un saut (phase de prise d'impulsion). Le patient présente immédiatement une impotence fonctionnelle totale du membre inférieur gauche. Il existe une notion de maladie d'Osgood-Schlatter bilatérale évoluant depuis plusieurs mois. L'examen clinique de ce genou retrouve un flessum avec un épanchement intra-articulaire ainsi qu'une douleur exquise de la tubérosité tibiale antérieure (TTA) reproduite à la palpation et à la contraction du quadriceps. Le cliché radiographique de profil du genou objective une fracture-arrachement déplacée de la TTA (Fig. 1). Le patient bénéficie d'une ostéosynthèse à foyer ouvert par vissage de la TTA au cours de laquelle l'exploration articulaire ne retrouve aucune lésion associée. Cette pathologie traumatique rare atteint essentiellement les adolescents sportifs en fin de croissance (lors de la période de pré-ossification) avec une nette prédominance masculine [1,2]. Classiquement, le mécanisme lésionnel est une violente contraction excentrique du quadriceps. Un antécédent de maladie d'Osgood-Schlatter ne précède pas nécessairement la fracture de la TTA mais il est fréquemment retrouvé. La classification qui fait référence concernant cette avulsion apophysaire est celle définie par Ogden ; elle repose sur l'analyse de la radiographie du genou de profil [1]. Les radiographies standard sont souvent suffisantes pour le diagnostic. Néanmoins, une IRM est parfois nécessaire à la recherche d'une atteinte méniscale ou ligamentaire associée [2]. Le traitement chirurgical est systématique pour tous les arrachements déplacés afin de permettre la restauration anatomique et fonctionnelle du genou $[1,2]$.

\section{Références}

1. Ogden JA, Tross RB, Murphy MJ (1980) Fractures of the tibial tuberosity in adolescents. J Bone Joint Surg Am 62:205-15

2. McKoy BE, Stanitski CL (2003) Acute tibial tubercle avulsion fractures. Orthop Clin N Am 34:397-403 